\title{
Implicit Runge-Kutta Methods of Optimal Order for Volterra Integro-Differential Equations
}

\author{
By Hermann Brunner
}

\begin{abstract}
Implicit Runge-Kutta methods with $m$ stages and optimal order $p=2 m$ for the approximate solution of Volterra integro-differential equations can be viewed as fully discretized collocation methods in certain polynomial spline spaces. The choice of the quadrature formulas needed for the full discretization of the collocations is investigated, and it is shown that, in contrast to ordinary differential equations, there exist (for fixed $m$ ) several optimal methods.
\end{abstract}

1. Introduction. In this paper we shall be concerned with the approximate solution of the initial-value problem for a nonlinear (first-order) Volterra integro-differential equation,

$$
y^{\prime}(t)=f(t, y(t))+\int_{t_{0}}^{t} k(t, s, y(s)) d s, \quad t \in I:=\left[t_{0}, T\right], y\left(t_{0}\right)=y_{0},
$$

with $f=f(t, y)$ and $k=k(t, s, y)$ being continuous on their respective domains $I \times \mathbf{R}$ and $S \times \mathbf{R}\left(S:=\left\{(t, s): t_{0} \leqq s \leqq t \leqq T\right\}\right)$ and such that (1:1) possesses a unique solution $y \in C^{\prime}(I)$. Even though the problem is frequently stated in a somewhat more general form,

$$
y^{\prime}(t)=F\left(t, y(t), \int_{t_{0}}^{t} k(t, s, y(s)) d s\right), \quad t \in I, y\left(t_{0}\right)=y_{0},
$$

we prefer dealing with the form (1.1) since it will make, above all, the connection with the corresponding initial-value problem for the ordinary differential equation, $y^{\prime}(t)=f(t, y(t)), y\left(t_{0}\right)=y_{0}$ (i.e. $\left.k(t, s, y) \equiv 0\right)$, more transparent. Furthermore, the analysis of the convergence properties of any numerical method for (1.1) (or (1.2)) will necessarily involve the linearization of the given equation and lead to a problem of the form

$$
y^{\prime}(t)=p(t) y(t)+q(t)+\int_{t_{0}}^{t} K(t, s) y(s) d s, \quad t \in I, y\left(t_{0}\right)=y_{0},
$$

where $p$ and $K$ represent, respectively, the partial derivatives $\partial f(t, y) / \partial y$ and $\partial k(t, s, y) / \partial y$ (or $\partial F(t, y, z) / \partial y$ and $F(t, y, z) / \partial z$ ), evaluated at suitable arguments (determined by the Mean-Value Theorem).

Received November 29, 1982.

1980 Mathematics Subject Classification. Primary 65R99, 45L10; Secondary 45J05.

Key words and phrases. Volterra integro-differential equations, collocation methods, implicit RungeKutta methods of optimal order. 
The given initial-value problem (1.1) will be solved numerically by implicit Runge-Kutta methods which, for a given fixed number of stages (denoted by $m \geqq 1$ ), have optimal order of convergence, $p=2 m$, at the mesh-points $\left\{t_{n}=t_{0}+n h\right.$ $\left.\left(n=1, \ldots, N: t_{N}=T\right)\right\}$. For the special case $k(t, s, y) \equiv 0$ these optimal methods are, of course, well known: for a given $m$ there exists a unique implicit Runge-Kutta method of order $p=2 m$ which can be viewed as a collocation method (based on the Gauss points) in a certain polynomial spline space (compare. e.g., Guillou et Soulé [8]. Norsett and Wanner [15]. Norsett [14] for studies of this aspect). However, if the integral operator is present in (1.1) (i.e. $k(t, s, y) \neq 0$ ), there is as yet no analogous analysis or list of such optimal Runge-Kutta methods. Nonoptimal methods have been discussed by various authors: Lubich [10], [11] presents the general theory concerning the structure of the order conditions of Runge-Kutta methods for (1.2) and lists a number of specific explicit methods: the question on the attainable order of implicit Runge-Kutta methods is not touched. Related methods, namely so-called block-by-block methods, have recently been investigated by Makroglou [12]; in addition. compare also the papers of Mocarsky [13]. Brunner [2]. Brunner and Lambert [4. pp. 84-87], and Feldstein and Sopka [6] for other step-by-step and Runge-Kutta methods. The survey article of Cryer [5, pp. 62-72] reviews the state of the art up to 1972 (the comprehensive bibliography includes the important contributions of Pouzet $(1960,1962)$ and of Tavernini (1971)). Finally, we should also like to mention the more recent survey paper of Baker [1].

It will be shown below that collocation in certain polynomial spline spaces (which will be subspaces of $C(I))$ is the natural setting for the analysis and the construction of implicit Runge-Kutta methods of optimal order for (1.1): while the resulting collocation equations are, in contrast to those for the case $k(t, s, y) \equiv 0$, not yet fully discretized (due to the presence of the integral operator), they furnish already the necessary information on the attainable order at the nodes of the approximating polynomial spline. Full discretization is then arrived at by selecting suitable quadrature formulas to approximate the integral operator; this discretization step may be interpreted as a perturbation of the collocation equations, with the perturbation terms being given by the quadrature errors. The corresponding perturbation analysis will then tell us how to choose the quadrature formulas in order to match the (already known) attainable optimal order of convergence at the nodes.

The polynomial spline space used for the approximation of the exact solution of (1.1) is defined as follows: let $N \geqq 1, m \geqq 1$ (with $N, m \in \mathbf{N}$ ), $t_{0}<t_{1}<\cdots<t_{N}=$ $T, Z_{N}:=\left\{t_{n}: n=1, \ldots, N-1\right\}, \bar{Z}_{N}:=Z_{N} \cup T$. and set $\sigma_{n}:=\left[t_{n}, t_{n+1}\right](n=0, \ldots$ $N-1)$. Then

$$
S_{m}^{(0)}\left(Z_{N}\right):=\left\{u \in C(I):\left.u\right|_{\sigma_{n}}=: u_{n} \in \pi_{m}(n=0, \ldots N-1)\right\},
$$

with $\operatorname{dim} S_{m}^{(0)}\left(Z_{N}\right)=N m+1$, is our approximating polynomial spline space. Let $X(N):=\bigcup_{n=0}^{N-1} X_{n}$, with

$$
X_{n}:=\left\{t_{n}+c_{i} h: 0 \leqq c_{1}<\cdots<c_{m} \leqq 1\right\} \quad(n=0, \ldots, N-1),
$$

denote the set of collocation points at which the desired approximation $u \in S_{m}^{(0)}\left(Z_{N}\right)$ is to satisfy the given integro-differential equation (1.1). This approximation is thus 
determined recursively by

$$
\begin{aligned}
u_{n}^{\prime}\left(t_{n}+c_{i} h\right)= & f\left(t_{n}+c_{i} h, u_{n}\left(t_{n}+c_{i} h\right)\right)+\int_{t_{n}}^{t_{n}+c_{i} h} k\left(t_{n}+c_{i} h, s, u_{n}(s)\right) d s \\
& +\sum_{k=0}^{n-1} \int_{t_{k}}^{t_{k+1}} k\left(t_{n}+c_{i} h, s, u_{k}(s)\right) d s, \\
& i=1, \ldots, m(n=0, \ldots, N-1) .
\end{aligned}
$$

Since $u \in C(I)$, we have

$$
u_{n}\left(t_{n}\right)=u_{n-1}\left(t_{n}\right), \quad t_{n} \in Z_{N}\left(\text { with } u_{0}\left(t_{0}\right)=y_{0}\right)
$$

In Section 2 we shall analyze the order of convergence, $p$, which is attainable globally (i.e. on $I$ ) and locally (on $\bar{Z}_{N}$ ) by the solution of (1.6). If the integrals in (1.6) over the subintervals $\left\{\left[t_{n}, t_{n}+c_{i} h\right]: i=1, \ldots, m\right\}$ and $\left\{\left[t_{k}, t_{k+1}\right]: 0 \leqq k \leqq\right.$ $n-1)$ are approximated by numerical quadrature (leading to the fully discretized collocation equations), we get a class of implicit Runge-Kutta methods for (1.1). The structure of these methods, together with their global and local convergence properties, will be studied in Section 3; this perturbation analysis will reveal how implicit Runge-Kutta methods of optimal order $p=2 m$ can be found (it will also show that there are several such optimal methods, in contrast to the case of an ordinary differential equation where there is precisely one implicit Runge-Kutta method with $p=2 m$ ). Finally, in Section 4 we give a number of specific optimal Runge-Kutta methods.

\section{The Attainable Order of Collocation Approximations.}

THEOREM 2.1. Suppose that the Volterra integro-differential equation (1.1) possesses a unique solution $y \in C^{m+1}(I)$; in particular, let $|\partial f(t, y) / \partial y| \leqq P,|\partial k(t, s, y) / \partial y|$ $\leqq M$, uniformly on $I \times \mathbf{R}$ and on $S \times \mathbf{R}$, respectively. Let $h>0$ in $t_{n}=t_{0}+\boldsymbol{n h}$ $\left(n=0, \ldots, N-1 ; t_{N}=T\right)$ be sufficiently small so that (1.6) defines a unique approximation $u \in S_{m}^{(0)}\left(Z_{N}\right)$ for any choice of the collocation parameters $\left\{c_{i}\right\}$ with $0 \leqq c_{1}<\cdots<c_{m} \leqq 1$. The error $e(t):=y(t)-u(t)$ then satisfies

$$
\begin{aligned}
& \|e\|_{\infty}:=\max \{|e(t)|: t \in I\}=\mathcal{O}\left(h^{m}\right), \\
& \left\|e^{\prime}\right\|_{\infty}:=\sup \left\{\left|e_{n}^{\prime}(t)\right|: t \in\left(t_{n}, t_{n+1}\right](n=0, \ldots, N-1)\right\}=\mathcal{O}\left(h^{m}\right),
\end{aligned}
$$

as $h \rightarrow 0_{+}, N h=T-t_{0}$.

Without loss of generality we have restricted our discussion to uniform partitions of $I$ : the generalization to so-called quasi-uniform partitions (sometimes also called $\gamma$-quasi-uniform partitions), where the quantities

$$
H_{N}^{\prime}:=\min \left\{t_{n+1}-t_{n}: 0 \leqq n \leqq N-1\right\}, \quad H_{N}^{\prime \prime}:=\max \left\{t_{n+1}-t_{n}: 0 \leqq n \leqq N-1\right\},
$$

satisfy $H_{N}^{\prime \prime} / H_{N}^{\prime}<\gamma<\infty$ for all $N$, is straightforward. We also note that the special choice $c_{1}=0$ and $c_{m}=1(m \geqslant 2)$ implies that $u \in C^{1}(I)$. 
Proof. It follows from (1.1) (with $t=t_{n}+c_{i} h$ ) and from (1.6) that

$$
\begin{aligned}
e_{n}^{\prime}\left(t_{n}+c_{i} h\right)= & f_{y}\left(t_{n}+c_{i} h, \cdot\right) \cdot e_{n}\left(t_{n}+c_{i} h\right) \\
& +h \int_{0}^{c_{i}} k_{y}\left(t_{n}+c_{i} h, t_{n}+\tau h, \cdot\right) \cdot e_{n}\left(t_{n}+\tau h\right) d \tau \\
& +h \sum_{k=0}^{n-1} \int_{0}^{1} k_{y}\left(t_{n}+c_{i} h, t_{k}+\tau h, \cdot\right) \cdot e_{k}\left(t_{k}+\tau h\right) d \tau
\end{aligned}
$$

$$
(i=1, \ldots, m)
$$

where $e_{n}:=\left.e\right|_{\sigma_{n}}$, and where the unspecified arguments in $f_{y}$ and $k_{y}$ are appropriate intermediate values given by the Mean-Value Theorem. Let us define

$$
p_{n, i}:=f_{v}\left(t_{n}+c_{i} h, \cdot\right), \quad K_{n, i}\left(t_{k}+\tau h\right):=k_{y}\left(t_{n}+c_{i} h, t_{k}+\tau h, \cdot\right) .
$$

Since $y \in C^{m+1}(I)$, we may write

$$
e_{n}\left(t_{n}+\tau h\right)=h^{m}\left(\sum_{j=0}^{m} \beta_{n, j} \cdot \tau^{j}+h \cdot R_{n}(\tau)\right), \quad t_{n}+\tau h \in \sigma_{n},
$$

and

$$
\begin{aligned}
& e_{n}^{\prime}\left(t_{n}+\tau h\right)=h^{m-1}\left(\sum_{j=1}^{m} j \cdot \beta_{n, j} \cdot \tau^{j-1}+h \cdot R_{n}^{\prime}(\tau)\right), \\
& t_{n}+\tau h \in\left(t_{n}, t_{n+1}\right] \quad(0 \leqq n \leqq N-1) ;
\end{aligned}
$$

here, $h^{m} \cdot \beta_{n, j}:=c_{n, j}-\alpha_{n, j}(j=0, \ldots, m)$, with $c_{n, j}:=h^{j} y^{(j)}\left(t_{n}\right) / j !, u_{n}\left(t_{n}+\tau h\right)$ $:=\sum_{j=0}^{m} \alpha_{n, j} \tau^{j}$, and

(2.4) $\quad R_{n}(\tau):=y^{(m+1)}\left(\xi_{n}\right) \cdot \tau^{m+1} /(m+1) !, \quad R_{n}^{\prime}(\tau):=y^{(m+1)}\left(\eta_{n}\right) \cdot \tau^{m} / m !$

$$
\left(t_{n}<\xi_{n}<t_{n}+\tau h, t_{n}<\eta_{n}<t_{n}+\tau h\right) .
$$

Hence, substitution of the above abbreviations and expressions in (2.1) yields the linear recurrence relation for $\beta_{n, 0}$ and the components of the vector $\beta_{n}:=$ $\left(\beta_{n, 1} \cdots \beta_{n, m}\right)^{r} \in \mathbf{R}^{m}(0 \leqq n \leqq N-1)$,

$$
\begin{aligned}
\sum_{j=1}^{m}(j & \left.\cdot c_{i}^{j-1}-h \cdot p_{n, i} \cdot c_{i}^{j}-h^{2} \cdot \int_{0}^{c_{i}} K_{n, i}\left(t_{n}+\tau h\right) \cdot \tau^{j} d \tau\right) \cdot \beta_{n, j} \\
= & \left(h \cdot p_{n, i}+h^{2} \cdot \int_{0}^{c_{1}} K_{n, i}\left(t_{n}+\tau h\right) d \tau\right) \cdot \beta_{n, 0} \\
& +h^{2} \sum_{k=1}^{n-1} \int_{0}^{1} K_{n, i}\left(t_{k}+\tau h\right) d \tau \cdot \beta_{k, 0} \\
& +h^{2} \sum_{k=0}^{n-1} \sum_{j=1}^{m} \int_{0}^{1} K_{n, i}\left(t_{k}+\tau h\right) \cdot \tau^{j} d \tau \cdot \beta_{k, j}+q_{n, i} \quad(i=1, \ldots, m),
\end{aligned}
$$

with

$$
\begin{aligned}
q_{n, i}:= & -h \cdot R_{n}^{\prime}\left(c_{i}\right)+h^{2} p_{n, i} \cdot R_{n}\left(c_{i}\right)+h^{3} \cdot \int_{0}^{c_{i}} K_{n, i}\left(t_{n}+\tau h\right) \cdot R_{n}(\tau) d \tau \\
& +h^{3} \sum_{k=0}^{n-1} \int_{0}^{1} K_{n, i}\left(t_{k}+\tau h\right) \cdot R_{k}(\tau) d \tau
\end{aligned}
$$


We have also used the fact that $\beta_{0,0}=0$, since $\beta_{0,0}=c_{0,0}-\alpha_{0,0}=y\left(t_{0}\right)-u_{0}\left(t_{0}\right)=$ 0 . Furthermore, it follows from $e \in C(I)$ that

$$
\beta_{k, 0}=\beta_{0,0}+\sum_{l=0}^{k-1}\left(\sum_{j=1}^{m} \beta_{l, j}+h \cdot R_{l}(1)\right), \quad \beta_{0,0}=0 \quad(1 \leqq k \leqq N-1) .
$$

If we now use this relation to express $\beta_{n, 0}$ and $\beta_{k, 0}(k<n)$ on the right-hand side of (2.5) in terms of the components of the vectors $\beta_{k}$, we arrive at

$$
\begin{aligned}
\sum_{j=1}^{m}(j & \left.\cdot c_{i}^{j-1}-h p_{n, i} c_{i}^{j}-h^{2} \cdot \int_{0}^{c_{i}} K_{n, i}\left(t_{n}+\tau h\right) \cdot \tau^{j} d \tau\right) \cdot \beta_{n, j} \\
= & h^{2} \sum_{k=0}^{n-1} \sum_{j=1}^{m} \beta_{k, j} \cdot \int_{0}^{1} K_{n, i}\left(t_{k}+\tau h\right) \cdot \tau^{j} d \tau \\
& +\left(p_{n, i}+h \cdot \int_{0}^{c_{i}} K_{n, i}\left(t_{n}+\tau h\right) d \tau\right) \cdot\left(h \sum_{k=0}^{n-1} \sum_{j=1}^{m} \beta_{k, j}\right) \\
& +h^{2} \sum_{k=1}^{n-1}\left(\sum_{l=0}^{k-1} \sum_{j=1}^{m} \beta_{l, j}\right) \cdot \int_{0}^{1} K_{n, i}\left(t_{k}+\tau h\right) d \tau+\tilde{q}_{n, i},
\end{aligned}
$$

where we have set

$$
\begin{aligned}
\tilde{q}_{n, i}:= & q_{n, i}+\left(p_{n, i}+h \cdot \int_{0}^{c_{i}} K_{n, i}\left(t_{n}+\tau h\right) d \tau\right) \cdot h^{2} \cdot \sum_{k=0}^{n-1} R_{k}(1) \\
& +h^{3} \sum_{k=1}^{n-1} \sum_{l=0}^{k-1} R_{l}(1) \cdot \int_{0}^{1} K_{n, i}\left(t_{k}+\tau h\right) d \tau,
\end{aligned}
$$

with $q_{n, i}$ given in (2.6).

We shall now show that $\beta_{n, 0}$ and the vectors $\beta_{n}$ satisfy

$$
\left\|\beta_{n}\right\|_{1}:=\sum_{j=1}^{m}\left|\beta_{n, j}\right| \leqslant h \cdot B,
$$

According to (2.2) and (2.3) this will then directly imply the two convergence statements of Theorem 2.1 .

We first note that the matrix defined by the coefficients of $\left\{\beta_{n, j}\right\}$ on the left-hand side of (2.8) is invertible whenever $h>0$ is sufficiently small: this follows from the fact that $\left|p_{n, i}\right| \leqq P,\left|K_{n, i}\left(t_{n}+\tau h\right)\right| \leqq M$, and the observation that, for $h=0$, the determinant of this matrix is essentially a Vandermonde determinant with value $m ! \Pi_{i>j}\left(c_{i}-c_{j}\right)$. Thus, let $\bar{h}>0$ be such that the $l_{1}$-norm of the inverse of this matrix is uniformly bounded by $G, h \in(0, \bar{h})(n=0, \ldots, N-1)$. Consider next the matrices formed by the coefficients of the vectors $\beta_{k}(k<n)$ in the first term on the right-hand side of (2.8): by our hypothesis on $k_{y}$ the $l_{\mathrm{i}}$-norm of each of these matrices is bounded by $m M$. Analogous arguments yield uniform bounds for the $l_{1}$-norms of the vectors occurring in the second and third term. Finally, according to (2.4) and the assumptions on the boundedness and the smoothness of $f_{y}, k_{y}$, and $y$, there is a finite constant $Q^{\prime}$ such that the vectors $q_{n}:=\left(q_{n, 1} \cdots q_{n, m}\right)^{T} \in \mathbf{R}^{m}$ 
$(0 \leqq n \leqq N-1)$ satisfy $\left\|q_{n}\right\|_{1} \leqq h Q^{\prime}$. In summary, (2.8) leads to the inequality

$$
\begin{array}{r}
\left\|\beta_{n}\right\|_{1} \leqq h C_{0}^{\prime} \sum_{k=0}^{n-1}\left\|\beta_{k}\right\|_{1}+h^{2} C_{1}^{\prime} \sum_{k=0}^{n-1}\left\|\beta_{k}\right\|_{1}+h C_{2}^{\prime}\left(h \cdot \sum_{k=1}^{n-1} \sum_{l=0}^{k-1}\left\|\beta_{l}\right\|_{1}\right)+h Q \\
(n=0, \ldots, N-1 ; h \in(0, \bar{h})),
\end{array}
$$

where $C_{0}^{\prime}:=m G M, C_{1}^{\prime}:=G(P+h M), C_{2}^{\prime}:=G M, Q:=G Q^{\prime}$. The right-hand side of (2.12) can be simplified as follows: since

$$
\begin{aligned}
h^{2} \sum_{k=1}^{n-1} \sum_{l=0}^{k-1}\left\|\beta_{l}\right\|_{1} & =h^{2} \sum_{k=0}^{n-2}(n-k-1) \cdot\left\|\beta_{k}\right\|_{1} \leqq h^{2} N \sum_{k=0}^{n-2}\left\|\beta_{k}\right\|_{1} \\
& \leqq h\left(T-t_{0}\right) \sum_{k=0}^{n-1}\left\|\beta_{k}\right\|_{1},
\end{aligned}
$$

we have

$$
\left\|\beta_{n}\right\|_{1} \leqq h C \sum_{k=0}^{n-1}\left\|\beta_{k}\right\|_{1}+h Q \quad(n=0, \ldots, N-1)
$$

where $C:=C_{0}^{\prime}+h C_{1}^{\prime}+C_{2}^{\prime}\left(T-t_{0}\right)$. This is the well-known discrete Gronwall inequality which yields the uniform bound

$$
\begin{aligned}
\left\|\beta_{n}\right\|_{1} \leqq h Q \cdot(1+h C) \cdot \exp \left(C\left(T-t_{0}\right)\right) & =: h B \\
(n & =0, \ldots, N-1 ; h \in(0, \bar{h})) .
\end{aligned}
$$

Furthermore, (2.7) leads to

$$
\left|\beta_{n, 0}\right| \leqq \sum_{k=0}^{n-1}\left(\left\|\beta_{k}\right\|_{1}+h M_{m+1}\right) \quad\left(M_{m+1}:=\max \left\{\left|y^{(m+1)}(t)\right| /(m+1) !: t \in I\right\}\right) \text {. }
$$

By (2.13) this becomes

$$
\begin{aligned}
\left|\beta_{n, 0}\right| \leqq N \cdot h B+N \cdot h M_{m+1}=\left(T-t_{0}\right) \cdot\left(B+M_{m+1}\right):=B^{\prime} \\
(n=1, \ldots, N-1) .
\end{aligned}
$$

We have thus verified the uniform bounds mentioned in (2.10), (2.11). To complete the proof we return to (2.2) and (2.3): from (2.2) we find

$$
\begin{aligned}
&\left|e_{n}\left(t_{n}+\tau h\right)\right| \leqq h^{m}\left(\left|\beta_{n, 0}\right|\right.\left.+\left\|\beta_{n}\right\|_{1}+h M_{m+1}\right) \leqq h^{m}\left(B^{\prime}+h B+h M_{m+1}\right) \\
&\left(t_{n}+\tau h \in \sigma_{n}, n=0, \ldots, N-1 ; h \rightarrow 0_{+}, N h=T-t_{0}\right),
\end{aligned}
$$

while (2.3) furnishes, in an analogous fashion,

$$
\begin{aligned}
\left|e_{n}^{\prime}\left(t_{n}+\tau h\right)\right| & \leqq h^{m-1}\left(m \cdot\left\|\beta_{n}\right\|_{1}+h \cdot m M_{m+1}\right) \\
& \leqq h^{m-1}\left(h \cdot m B+h \cdot m M_{m+1}\right)=h^{m} \cdot m\left(B+M_{m+1}\right) .
\end{aligned}
$$

The above reasoning also makes it clear that the exponent $m$ of $h$ in the upper bounds for $\|e\|_{\infty}$ and $\left\|e^{\prime}\right\|_{\infty}$ is best possible, i.e. it cannot be replaced by $(m+1)$.

While every choice of the collocation parameters $\left\{c_{i}\right\}$ leads to the same global convergence rate $p=m$, there exist special sets of these parameters for which one obtains a higher order of convergence at the nodes of the approximating polynomial spline. This is made precise in 
THEOREM 2.2. Suppose that the solution of (1.1) satisfies $y \in C^{2 m-v}(I)$, where $\nu \in\{0,1,2\}$, and let $f_{y}$ and $k_{y}$ be subject to the hypotheses stated in Theorem 2.1.

(a) If $\nu=0$, and if $u \in S_{m}^{(0)}\left(Z_{N}\right)$ denotes the collocation approximation determined by (1.6), where the collocation parameters are the zeros of $P_{m}(2 s-1)($ Gauss points on $(0,1))$, then

$$
e\left(t_{n}\right)=\vartheta\left(h^{2 m}\right) \quad \text { for } t_{n} \in \bar{Z}_{N}\left(h \rightarrow 0_{+}, N h=T-t_{0}\right) .
$$

(b) If $\nu=1$, and if $u \in S_{m}^{(0)}\left(Z_{N}\right)$ denotes the collocation approximation determined by (1.6), where the collocation parameters $\left\{c_{i}\right\}$ are the zeros of $s \cdot P_{m-1}^{(0,1)}(2 s-1)$, or of $(s-1) \cdot P_{m-1}^{(1,0)}(2 s-1)$ (Radau points for $[0,1)$ and $(0,1]$, respectively), then

$$
e\left(t_{n}\right)=\theta\left(h^{2 m-1}\right) \text { for } t_{n} \in \bar{Z}_{N}\left(h \rightarrow 0_{+}, N h=T-t_{0}\right) .
$$

(c) If $\nu=2$, and if $u \in S_{m}^{(0)}\left(Z_{N}\right)$ denotes the collocation approximation determined by (1.6), where the collocation parameters $\left\{c_{i}\right\}$ are the zeros of $s(s-1) \cdot P_{m-2}^{(1,1)}(2 s-1)$ (Lobatto points for $[0,1])$, then

$$
e\left(t_{n}\right)=\mathcal{O}\left(h^{2 m-2}\right) \text { for } t_{n} \in \bar{Z}_{N}\left(h \rightarrow 0_{+}, N h=T-t_{0}\right) .
$$

(Note that $c_{1}=0$ and $c_{m}=1$ imply that $u$ is not only in $C(I)$ but also in $C^{1}(I)$.)

For a comprehensive analysis of the quadrature formulas of Gauss, Radau, and Lobatto (including a study of the Jacobi polynomials $P_{m}^{(\alpha, \beta)}(t)$ ) we refer the reader to Ghizzetti and Ossicini [7, pp. 58-68 and pp. 92-110].

Proof. According to (1.6) the collocation approximation $u \in S_{m}^{(0)}\left(Z_{N}\right)$ satisfies

$$
u^{\prime}(t)=f(t, u(t))+\int_{t_{0}}^{t} k(t, s, u(s)) d s-\delta(t), \quad t \in I,
$$

with $\delta\left(t_{0}\right)=0$; here, the defect $\delta(t)$ vanishes on the set $X(N)$ of collocation points (recall (1.5)). If we now subtract (1.7) from (1.1) we are led to

$$
e^{\prime}(t)=p(t) \cdot e(t)+\delta(t)+\int_{t_{0}}^{t} K(t, s) \cdot e(s) d s, \quad t \in I, e\left(t_{0}\right)=0
$$

In analogy to the proof of Theorem 2.1 we have set $p(t):=f_{y}(t, \cdot)$ and $K(t, s):=k_{y}(t, s, \cdot)$, with suitable last arguments given by the Mean-Value Theorem. The solution of this linear Volterra integro-differential equation is given in terms of the resolvent kernel $R=R(t, s)$ : since such an integro-differential equation can be written as a Volterra integral equation of the second kind, $R(t, s)$ is the resolvent kernel of the kernel of this second-kind equation, $Q(t, s):=p(s)+$ $\int_{s}^{t} K(v, s) d v,(t, s) \in S$. We thus obtain (see also, e.g. [3, pp. 264-265])

$$
e(t)=R\left(t, t_{0}\right) \cdot e\left(t_{0}\right)+\int_{t_{0}}^{t} R(t, s) \cdot \delta(s) d s, \quad t \in I, e\left(t_{0}\right)=0
$$

The assumed smoothness of $p$ and $K$ is inherited by $Q$ and hence by $R$, while the defect $\delta$ is piecewise smooth on $I$ (recall (2.17)). Now let $t=t_{n} \in \bar{Z}_{N}$ in (2.19), and let $\left\{d_{i}\right\}$ be $m$ given points in $[0,1], 0 \leqq d_{1}<\cdots<d_{m} \leqq 1$. We may then write

$$
\begin{aligned}
e\left(t_{n}\right) & =h \cdot \sum_{k=0}^{n-1} \int_{0}^{1} R\left(t_{n}, t_{k}+\tau h\right) \cdot \delta\left(t_{k}+\tau h\right) d \tau \\
& =h \sum_{k=0}^{n-1}\left(\sum_{j=1}^{m} w_{j} R\left(t_{n}, t_{k}+d_{j} h\right) \cdot \delta\left(t_{k}+d_{j} h\right)+E_{n, k}\right),
\end{aligned}
$$


where $E_{n, k}$ denotes the error term associated with the quadrature formula based on the abscissas $\left\{t_{k}+d_{j} h\right\}$ and the weights $\left\{w_{j}\right\}(j=1, \ldots, m)$. If we choose $d_{j}=c_{j}$ (the collocation parameters), then $\delta\left(t_{k}+c_{j} h\right)=0(j=1, \ldots, m ; 0 \leqq k \leqq N-1)$, and the above relation becomes

$$
e\left(t_{n}\right)=h \cdot \sum_{k=0}^{n \cdots 1} E_{n, k} \quad\left(t_{n} \in \bar{Z}_{N}\right) .
$$

Assuming that $E_{n, k}=\mathcal{O}\left(h^{r}\right)(0 \leqq k<n \leqq N-1)$, and observing that the number of terms in the above sum is at most equal to $N$, where $N h=T-t_{0}<\infty$, we have the result that the order of the error at the nodes is equal to the order of the quadrature formula defined by the parameters $\left\{c_{i}\right\}$ and the weights $\left\{w_{i}\right\}$. This result generalizes the analogous result for ordinary differential equations (i.e. (1.1) with $k(t, s, y) \equiv 0)$ derived in [8], [15]. The three particular results of Theorem 2.2 now follow immediately by observing that the error terms for the $m$-point quadrature formulas of Gauss, Radau, and Lobatto, contain, respectively, the derivatives of order $2 m, 2 m-1$, and $2 m-2$, of the integrand (see [7, pp. 92-110] for details). This also shows that the local order $p=2 m$ (for the collocation parameters being equal to the $m$ Gauss points in $(0,1))$ is optimal.

3. The Discretized Collocation Equations: Runge-Kutta Methods. In most cases the integrals occurring in the collocation equation (1.6) cannot be found analytically but have to be approximated by suitable quadrature formulas. This means that, instead of $u \in S_{m}^{(0)}\left(Z_{N}\right)$, we compute an approximation $\hat{u}=S_{m}^{(0)}\left(Z_{N}\right)$ from a perturbed collocation equation,

$$
\begin{aligned}
\hat{u}_{n}^{\prime}\left(t_{n}+c_{i} h\right)= & f\left(t_{n}+c_{i} h, \hat{u}_{n}\left(t_{n}+c_{i} h\right)\right) \\
& +h \sum_{j=1}^{\mu_{0}} w_{i j} \cdot k\left(t_{n}+c_{i} h, t_{n}+d_{i j} h, \hat{u}_{n}\left(t_{n}+d_{i j} h\right)\right) \\
& +h \sum_{k=0}^{n-1} \sum_{j=1}^{\mu_{1}} w_{j} \cdot k\left(t_{n}+c_{i} h, t_{k}+d_{j} h, \hat{u}_{k}\left(t_{k}+d_{j} h\right)\right), \\
& i=1, \ldots, m(n=0, \ldots, N-1),
\end{aligned}
$$

where $0 \leqq d_{i, 1}<\cdots<d_{i, \mu_{0}} \leqq c_{i}(i=1, \ldots, m), 0 \leqq d_{1}<\cdots<d_{\mu_{1}} \leqq 1$, with $\mu_{0}$ and $\mu_{1}$ denoting given positive integers. Since the given kernel in (1.1) is only defined for $s \leqq t$, we shall only study the natural discretizations using the quadrature abscissas $t_{n}+d_{i j} h \leqq t_{n}+c_{i} h\left(j=1, \ldots, \mu_{0}\right)$; see also the remark in Section 5 .

Let $\hat{e}(t):=y(t)-\hat{u}(t)$, and set $\varepsilon(t):=u(t)-\hat{u}(t)$. Since $\hat{e}(t)=(y(t)-u(t))+$ $(u(t)-\hat{u}(t))=e(t)+\varepsilon(t)$, we have

$$
\|\hat{e}\|_{\infty} \leqq\|e\|_{\infty}+\|\varepsilon\|_{\infty}
$$

and

$$
\left|\hat{e}\left(t_{n}\right)\right| \leqq\left|e\left(t_{n}\right)\right|+\left|\varepsilon\left(t_{n}\right)\right|, \quad t_{n} \in \bar{Z}_{N},
$$

where the (optimal) orders of the first terms on the right-hand sides of (3.2! 'nd (3.3) are given by Theorem 2.1 and Theorem 2.2. 
If we subtract the discretized collocation equation (3.1) from the exact collocation equation (1.6), we obtain

$$
\begin{aligned}
& \varepsilon_{n}^{\prime}\left(t_{n}+c_{i} h\right)= p_{n, i} \cdot \varepsilon_{n}\left(t_{n}+c_{i} h\right) \\
&+h\left\{\int_{0}^{c_{i}} k\left(t_{n}+c_{i} h, t_{n}+\tau h, u_{n}\left(t_{n}+\tau h\right)\right) d \tau\right. \\
&\left.\quad-\sum_{j=1}^{\mu_{0}} w_{i j} \cdot k\left(t_{n}+c_{i} h, t_{n}+d_{i j} h, \hat{u}_{n}\left(t_{n}+d_{i j} h\right)\right)\right\} \\
&++\sum_{k=0}^{n-1}\left\{\int_{0}^{1} k\left(t_{n}+c_{i} h, t_{k}+\tau h, u_{k}\left(t_{k}+\tau h\right)\right) d \tau\right. \\
&\left.-\sum_{j=1}^{\mu_{1}} w_{j} \cdot k\left(t_{n}+c_{i} h, t_{k}+d_{j} h, \hat{u}_{k}\left(t_{k}+d_{j} h\right)\right)\right\} \\
& \quad(i=1, \ldots, m),
\end{aligned}
$$

which we write in the form

$$
\varepsilon_{n}^{\prime}\left(t_{n}+c_{i} h\right)=p_{n, i} \cdot \varepsilon_{n}\left(t_{n}+c_{i} h\right)+h \cdot\left\{J_{n, i}^{(n)}-\hat{J}_{n, i}^{(n)}\right\}+h \sum_{k=0}^{n-1}\left\{J_{k, i}^{(n)}-\hat{J}_{k, i}^{(n)}\right\}
$$

Suppose that the quadrature error terms are

$$
\begin{aligned}
& E_{n, i}^{(n)}:=J_{n, i}^{(n)}-\hat{J}_{n, i}^{(n)} \quad(i=1, \ldots, m), \\
& E_{k, i}^{(n)}:=J_{k, i}^{(n)}-\hat{J}_{k, i}^{(n)} \quad(i=1, \ldots, m ; k<n) .
\end{aligned}
$$

THEOREM 3.1. Let the assumptions of Theorem 2.2 hold; suppose further that for all sufficiently smooth integrands we have

$$
\left|E_{n, i}^{(n)}\right| \leqq C_{0} h^{r_{0}}, \quad\left|E_{k, i}^{(n)}\right| \leqq C_{1} h^{r_{1}} \quad(k<n), i=1, \ldots, m,
$$

with $r:=\min \left(r_{0}+1, r_{1}\right)$. Then

$$
\|\varepsilon\|_{\infty}=\theta\left(h^{r}\right), \quad\left\|\varepsilon^{\prime}\right\|_{\infty}=\theta\left(h^{r}\right) .
$$

Proof. It follows from the definition of $\varepsilon_{n}(t):=u_{n}(t)-\hat{u}_{n}(t), t \in \sigma_{n}$, that $\varepsilon_{n} \in \pi_{m}$. We thus have

$$
\varepsilon_{n}\left(t_{n}+\tau h\right):=\sum_{l=0}^{m} \delta_{n, l} \tau^{l}, \quad t_{n}+\tau h \in \sigma_{n}, \text { with } \delta_{0,0}=0 .
$$

According to (3.6), (3.7) the error equation (3.4) may be written as

$$
\begin{aligned}
\varepsilon_{n}^{\prime}\left(t_{n}+c_{i} h\right)= & p_{n, i} \cdot \varepsilon_{n}\left(t_{n}+c_{i} h\right) \\
& +h \sum_{j=1}^{\mu_{0}} w_{i j} \cdot K_{n, i}\left(t_{n}+d_{i j} h\right) \cdot \varepsilon_{n}\left(t_{n}+d_{i j} h\right) \\
& +h \sum_{k=0}^{n-1} \sum_{j=1}^{\mu_{1}} w_{j} \cdot K_{n, i}\left(t_{k}+d_{j} h\right) \cdot \varepsilon_{k}\left(t_{k}+d_{j} h\right) \\
& +\left\{h E_{n, i}^{(n)}+h \sum_{k=0}^{n-1} E_{k, i}^{(n)}\right\},
\end{aligned}
$$


where $p_{n, i}$ and $K_{n, i}$ denote, as in Section 2, appropriate values of the partial derivatives $f_{y}$ and $k_{y}$. If we now substitute the expressions for $\varepsilon_{n}$ and for $\varepsilon_{n}^{\prime}$ in Eq. (3.10), we find, in complete analogy to (2.8).

$$
\begin{aligned}
\sum_{l=1}^{m}\left(l \cdot c_{i}^{l-1}-h p_{n, i} \cdot c_{i}^{l}-h^{2} \sum_{j=1}^{\mu_{0}} w_{i j} K_{n, i}\left(t_{n}+d_{i j} h\right) d_{i j}^{l}\right) \cdot \delta_{n, l} \\
=h^{2} \sum_{k=0}^{n-1} \sum_{l=1}^{m} \delta_{k, l}\left(\sum_{j=1}^{\mu_{1}} w_{j} K_{n, i}\left(t_{n}+d_{j} h\right) \cdot d_{j}^{l}\right) \\
+\left(p_{n, i}+h \sum_{j=1}^{\mu_{0}} w_{i j} K_{n, i}\left(t_{n}+d_{i j} h\right)\right) \cdot\left(h \sum_{k=0}^{n-1} \sum_{l=1}^{m} \delta_{k, l}\right) \\
+h^{2} \sum_{k=1}^{n-1} \sum_{j=1}^{m} w_{j} K_{n, i}\left(t_{k}+d_{j} h\right) \cdot\left(\sum_{\nu=0}^{k-1} \sum_{l=1}^{m} \delta_{v, l}\right)+h \cdot T_{n, i} \\
\quad(i=1, \ldots, m),
\end{aligned}
$$

where

$$
T_{n, i}:=h E_{n, i}^{(n)}+h \sum_{k=0}^{n-1} E_{k, i}^{(n)}
$$

In (3.11) we have made use of the relation

$$
\delta_{n, 0}=\delta_{0,0}+\sum_{k=0}^{n-1} \sum_{l=1}^{m} \delta_{k, l}, \quad \delta_{0,0}=0
$$

which is a consequence of the continuity requirement for $\varepsilon(t)$ at $t=t_{n} \in Z_{N}$ (recall also the analogous relation (2.7)).

Since the recurrence relation (3.11) has exactly the same structure as the recurrence relation (2.8), the proof is completed along the lines of the proof for Theorem 2.1. Let $T_{n}:=\left(T_{n, 1} \cdots T_{n, m}\right)^{T} \in \mathbf{R}^{m}$. It follows from (3.12), (3.8) that

$$
\begin{aligned}
\left\|T_{n}\right\|_{1} & \leqq C_{0} h^{r_{0}+1}+N h \cdot C_{1} h^{r_{1}}=h^{r}\left(C_{0} h^{r_{0}+1-r}+\left(T-t_{0}\right) C_{1} h^{r_{1}-r}\right) \\
& =: h^{r} Q^{\prime} \quad(n=0, \ldots, N-1) .
\end{aligned}
$$

Hence, setting $\delta_{n}:=\left(\delta_{n, 1} \cdots \delta_{n, m}\right)^{T} \in \mathbf{R}^{m}$, we find an inequality of the form

$$
\left\|\delta_{n}\right\|_{1} \leqslant h D_{0} \sum_{k=0}^{n-1}\left\|\delta_{k}\right\|_{1}+h \cdot h^{r} Q \quad(n=0, \ldots, N-1) ;
$$

here, we have assumed that the quadrature weights $\left\{w_{i j}\right\}$ and $\left\{w_{j}\right\}$ satisfy $\left|w_{i j}\right| \leqq W$, $\left|w_{j}\right| \leqq W$ (which holds, of course, for interpolatory quadrature formulas since the number of abscissas in the formulas used here is fixed). Applying the discrete Gronwall Lemma we arrive at

$$
\begin{aligned}
&\left\|\delta_{n}\right\|_{1} \leqq h \cdot Q h^{r} \cdot\left(1+h D_{0}\right) \cdot \exp \left(D_{0}\left(T-t_{0}\right)\right)=: h \cdot D h^{r} \\
&\left(n=0, \ldots, N-1 ; h \rightarrow 0_{+}, N h=T-t_{0}\right) .
\end{aligned}
$$

Now (3.13) implies the estimate

$$
\left|\delta_{n, 0}\right| \leqq \sum_{k=0}^{n-1}\left\|\delta_{k}\right\|_{1} \leqq N h \cdot D h^{r}=\left(T-t_{0}\right) \cdot D h^{r}=: D^{\prime} h^{r},
$$


which in turns allows us to obtain

$$
\left|\varepsilon_{n}\left(t_{n}+\tau h\right)\right| \leqq\left|\delta_{n, 0}\right|+\left\|\delta_{n}\right\|_{1} \leqq D^{\prime} h^{r}+h \cdot D h^{r}=\left(D^{\prime}+h D\right) h^{r},
$$

and

$$
\begin{aligned}
\left|\varepsilon_{n}^{\prime}\left(t_{n}+\tau h\right)\right| \leqq h^{-1} m \cdot\left\|\delta_{n}\right\|_{1} \leqq h^{-1} m h \cdot D h^{r}=m D h^{r} \\
\quad\left(n=0, \ldots, N-1 ; h \rightarrow 0_{+}, N h=T-t_{0}\right) .
\end{aligned}
$$

This completes the proof of Theorem 3.1.

COROLlaRY 3.1. Let the assumptions of Theorem 3.1 hold. Then

$$
\|\hat{e}\|_{\infty} \leqq Q_{0} h^{m}+Q_{1} h^{r} \quad\left(h \rightarrow 0_{+}, N h=T-t_{0}\right) .
$$

Furthermore, if $e\left(t_{n}\right)=\Theta\left(h^{p^{*}}\right)$ for $t_{n} \in \bar{Z}_{N}$, then

$$
\hat{e}\left(t_{n}\right)=\vartheta\left(h^{q}\right), \quad t_{n} \in \bar{Z}_{N}, \text { with } q:=\min \left(p^{*}, r\right) .
$$

In particular, if $P_{m}\left(2 c_{i}-1\right)=0(i=1, \ldots, m)$ (Gauss points), and if, in the perturbed collocation equation (3.1), we have $d_{j}=c_{j}, d_{i j}=c_{j} c_{i}\left(\right.$ with $\left.\mu_{0}=\mu_{1}=m\right)$, with the quadrature formulas being interpolatory, then

$$
\hat{e}\left(t_{n}\right)=\theta\left(h^{2 m}\right), \quad t_{n} \in \bar{Z}_{N} \text {. }
$$

Proof. The bound in (3.16) follows from (3.2), Theorem 2.1, and (3.9), while (3.17) is a consequence of (3.3), Theorem 2.2, and (3.9). Finally, if the quadrature formulas used in (3.1) and characterized by (3.6), (3.7) are $m$-point Gauss formulas, then we have $r_{0}=r_{1}=2 m$, and hence $r=2 m$. The result (3.18) is therefore implied by (2.14) and (3.9).

We note that approximations $\hat{u} \in S_{m}^{(0)}\left(Z_{N}\right)$ determined by (3.1) and satisfying (3.18) do also result if $r_{0}=2 m-1$ in (3.8), since $r=\min \left(r_{0}+1, r_{1}\right)=2 m$, provided the quadrature approximations $\hat{J}_{k, i}^{(n)}(k<n)$ in (3.4), (3.7) are still the $m$-point Gauss formulas (i.e. $\mu_{1}=m$ ). It is well known that the two Radau quadrature formulas described in (2.15) have this property, thus demonstrating the fact that there is no unique optimal method (i.e. a method for which (3.18) holds) for the solution of $(1.1)(k(t, s, y) \not \equiv 0)$. This will be elaborated in the following section, by means of specific examples of such methods.

We conclude this section by rewriting the discretized collocation equations (3.1) in a form which exhibits more clearly the fact that (3.1) defines a class of implicit Runge-Kutta methods for the solution of (1.1). Define $Y_{j}^{(n)}:=u_{n}^{\prime}\left(t_{n}+c_{i} h\right)$ (where $\left.u_{n}^{\prime} \in \pi_{m-1}\right)$, and set

$$
l_{j}(\tau):=\prod_{\substack{r=1 \\ r \neq j}}^{m} \frac{\tau-c_{r}}{c_{j}-c_{r}} \quad(j=1, \ldots, m) .
$$

Since $u_{n}^{\prime}\left(t_{n}+\tau h\right)=\sum_{j=1}^{m} l_{j}(\tau) \cdot Y_{j}^{(n)}$, we have

$$
u_{n}\left(t_{n}+\tau h\right)=y_{n}+h \sum_{j=1}^{m} \alpha_{j}(\tau) \cdot Y_{j}^{(n)},
$$

where we have set $y_{n}:=u_{n}\left(t_{n}\right)\left(=u_{n-1}\left(t_{n}\right)\right)$, and

$$
\alpha_{j}(\tau):=\int_{0}^{\tau} l_{j}(u) d u, \quad j=1, \ldots, m .
$$


If we let $a_{i j}:=\alpha_{j}\left(c_{i}\right)$, then $u_{n}\left(t_{n}+c_{i} h\right)=y_{n}+h \sum_{j=1}^{m} a_{i j} Y_{j}^{(n)}$. Thus, the collocation equations (1.6) may be written in the form

$$
\begin{aligned}
Y_{i}^{(n)}= & f\left(t_{n}+c_{i} h, y_{n}+h \sum_{j=1}^{m} a_{i j} Y_{j}^{(n)}\right) \\
& +h \cdot \int_{0}^{c_{i}} k\left(t_{n}+c_{i} h, t_{n}+\tau h, y_{n}+h \sum_{j=1}^{m} \alpha_{j}(\tau) \cdot Y_{j}^{(n)}\right) d \tau \\
& +h \cdot \sum_{k=0}^{n-1} \int_{0}^{1} k\left(t_{n}+c_{i} h, t_{k}+\tau h, y_{k}+h \sum_{j=1}^{m} \alpha_{j}(\tau) \cdot Y_{j}^{(k)}\right) d \tau \\
& (i=1, \ldots, m) .
\end{aligned}
$$

The approximate value at $t=t_{n+1}$ is then given by

$$
y_{n+1}=u_{n}\left(t_{n}+h\right)=y_{n}+h \sum_{i=1}^{m} b_{i} Y_{i}^{(n)}, \quad b_{i}:=\alpha_{i}(1)(n=0, \ldots, N-1) .
$$

The integrals $J_{k, i}^{(n)}$ introduced in (3.5) now become

$$
J_{k, i}^{(n)}:= \begin{cases}\int_{0}^{1} k\left(t_{n}+c_{i} h, t_{k}+\tau h, y_{k}+h \sum_{j=1}^{m} \alpha_{j}(\tau) Y_{j}^{(k)}\right) d \tau, & 0 \leqq k<n, \\ \int_{0}^{c_{1}} k\left(t_{n}+c_{i} h, t_{n}+\tau h, y_{n}+h \sum_{j=1}^{m} \alpha_{j}(\tau) Y_{j}^{(n)}\right) d \tau, & k=n \\ & (i=1, \ldots, m) .\end{cases}
$$

The fully discretized collocation equations (i.e. (3.1)) can now be written down in terms of expressions $\hat{Y}_{j}^{(k)}:=\hat{u}_{k}^{\prime}\left(t_{k}+c_{j} h\right)(k \leqq n)$; we have

$$
\begin{gathered}
\hat{Y}_{i}^{(n)}=f\left(t_{n}+c_{i} h, \hat{y}_{n}+h \sum_{j=1}^{m} a_{i j} \hat{Y}_{j}^{(n)}\right) \\
+h \hat{J}_{n, i}^{(n)}+h \sum_{k=0}^{n-1} \hat{J}_{k, i}^{(n)} \quad(i=1, \ldots, m), \\
\hat{y}_{n+1}=\hat{u}_{n}\left(t_{n}+h\right)=\hat{y}_{n}+h \sum_{i=1}^{m} b_{i} \hat{Y}_{i}^{(n)} \quad(n=0, \ldots, N-1),
\end{gathered}
$$

where

$$
\hat{J}_{k, i}^{(n)}:= \begin{cases}\sum_{l=1}^{\mu_{1}} w_{l} \cdot k\left(t_{n}+c_{i} h, t_{k}+d_{l} h, \hat{y}_{k}+h \sum_{j=1}^{m} \alpha_{j}\left(d_{l}\right) \cdot \hat{Y}_{j}^{(k)}\right), & 0 \leqq k<n, \\ \sum_{l=1}^{\mu_{0}} w_{i, l} \cdot k\left(t_{n}+c_{i} h, t_{n}+d_{i l} h, \hat{y}_{n}+h \sum_{j=1}^{m} \alpha_{j}\left(d_{i l}\right) \cdot \hat{Y}_{j}^{(n)}\right), & k=n\end{cases}
$$$$
(i=1, \ldots, m) .
$$ 
In the following we shall restrict our analysis to the case where

$$
\mu_{0}=\mu_{1}=m ; \quad d_{l}=c_{l} ; \quad d_{i l}=c_{l} \cdot c_{i} \quad(l=1, \ldots, m ; i=1, \ldots, m) .
$$

The following notation will be used:

$$
a_{l j}^{(i)}:=\alpha_{j}\left(c_{l} \cdot c_{i}\right) ; \quad \bar{a}_{i l}:=\int_{0}^{c_{i}} \lambda_{l}^{(i)}(\tau) d \tau,
$$

where

$$
\lambda_{l}^{(i)}(\tau):=c_{i}^{--m+1} \cdot \prod_{\substack{r=1 \\ r \neq 1}}^{m}\left(\tau-c_{i} \cdot c_{r}\right) /\left(c_{l}-c_{r}\right)
$$

denotes the $l$ th Lagrange fundamental polynomial for the points $\left\{c_{i} c_{1}, \ldots, c_{i} c_{m}\right\}$. Therefore, if we use $m$-point interpolatory quadrature in (3.1), based on the above abscissas, we find that (3.1) yields the following class of Runge-Kutta methods for the Volterra integro-differential equation (1.1):

$$
\begin{aligned}
\hat{Y}_{i}^{(n)}= & f\left(t_{n}+c_{i} h, \hat{y}_{n}+h \sum_{j=1}^{m} a_{i j} \hat{Y}_{j}^{(n)}\right) \\
& +h \sum_{l=1}^{m} \bar{a}_{i l} \cdot k\left(t_{n}+c_{i} h, t_{n}+c_{l} c_{i} h, \hat{y}_{n}+h \sum_{j=1}^{m} a_{l j}^{(i)} \cdot \hat{Y}_{j}^{(n)}\right) \\
& +h \sum_{k=0}^{n-1} \sum_{l=1}^{m} b_{l} \cdot k\left(t_{n}+c_{i} h, t_{k}+c_{l} h, \hat{y}_{k}+h \sum_{j=1}^{m} a_{l j} \cdot \hat{Y}_{j}^{(k)}\right) \\
& \quad(i=1, \ldots, m),
\end{aligned}
$$

with $\hat{y}_{n+1}$ given by (3.25).

4. Examples. We have seen that an implicit Runge-Kutta method (3.29) for (1.1) is characterized by the following arrays:

$$
\begin{gathered}
\left\{c_{1}, \ldots, c_{m}\right\} ; \quad\left\{a_{i j}: i, j=1, \ldots, m\right\} ; \quad\left\{b_{1}, \ldots, b_{m}\right\} ; \\
\left\{\bar{a}_{i l}: i, l=1, \ldots, m\right\} ; \quad\left\{a_{l j}^{(i)}: l, j=1, \ldots, m ; i=1, \ldots, m\right\} .
\end{gathered}
$$

In the following we shall illustrate the analysis of Section 3 by presenting the three optimal methods of order $p=2 m=4$.

Example 4.1. $m=2 ; c_{1}=(3-\sqrt{3}) / 6, c_{2}=(3+\sqrt{3}) / 6\left(\right.$ zeros of $\left.P_{2}(2 s-1)\right)$. Here we find the following parameters:

$$
\begin{gathered}
A:=\left(a_{i j}\right)=\left(\begin{array}{cc}
1 / 4 & (3-2 \sqrt{3}) / 12 \\
(3+2 \sqrt{3}) / 12 & 1 / 4
\end{array}\right) ; \quad b_{1}=b_{2}=1 / 2 ; \\
D:=\left(d_{i l}\right)=\left(\begin{array}{cc}
c_{1}^{2} & c_{1} c_{2} \\
c_{2} c_{1} & c_{2}^{2}
\end{array}\right)=\left(\begin{array}{cc}
(2-\sqrt{3}) / 6 & 1 / 6 \\
1 / 6 & (2+\sqrt{3}) / 6
\end{array}\right) ; \\
\bar{A}:=\left(\bar{a}_{i l}\right)=\left(\begin{array}{cc}
(3-\sqrt{3}) / 12 & (3-\sqrt{3}) / 12 \\
(3+\sqrt{3}) / 12 & (3+\sqrt{3}) / 12
\end{array}\right) ; \\
a_{11}^{(1)}=(6-\sqrt{3}) / 72, \quad a_{12}^{(1)}=(18-11 \sqrt{3}) / 72 \\
a_{21}^{(1)}=(6+5 \sqrt{3}) / 72, \quad a_{22}^{(1)}=(6-5 \sqrt{3}) / 72
\end{gathered}
$$




$$
\begin{array}{ll}
a_{11}^{(2)}=(6+5 \sqrt{3}) / 72, & a_{12}^{(2)}=(6-5 \sqrt{3}) / 72, \\
a_{21}^{(2)}=(18+11 \sqrt{3}) / 72, & a_{22}^{(2)}=(6+\sqrt{3}) / 72 .
\end{array}
$$

Here, $r_{0}=r_{1}=2 m=4$, and hence $r=4$.

Example 4.2. $m=2 ; c_{1}=(3-\sqrt{3}) / 6, c_{2}=(3+\sqrt{3}) / 6 ; \tilde{c}_{1}=0, \tilde{c}_{2}=2 / 3(\operatorname{Radau}$ points for $[0,1)) ; d_{i l}=c_{i} \tilde{c}_{l}$.

The Runge-Kutta parameters are:

$A, b_{1}$ and $b_{2}$ as in Example 4.1;

$$
\begin{aligned}
& D=\left(d_{i l}\right)=\left(\begin{array}{ll}
c_{1} \tilde{c}_{1} & c_{1} \tilde{c}_{2} \\
c_{2} \tilde{c}_{1} & c_{2} \tilde{c}_{2}
\end{array}\right)=\left(\begin{array}{ll}
0 & (3-\sqrt{3}) / 9 \\
0 & (3+\sqrt{3}) / 9
\end{array}\right) \\
& \bar{A}:=\left(\bar{a}_{i l}\right)=\left(\begin{array}{ll}
(3-\sqrt{3}) / 24 & (3-\sqrt{3}) / 8 \\
(3+\sqrt{3}) / 24 & (3+\sqrt{3}) / 8
\end{array}\right) ; \\
& a_{11}^{(1)}=0, \quad a_{12}^{(1)}=0, \quad a_{21}^{(1)}=(3+\sqrt{3}) / 27, \quad a_{22}^{(1)}=(6-4 \sqrt{3}) / 27 \text {; } \\
& a_{11}^{(2)}=0, \quad a_{12}^{(2)}=0, \quad a_{21}^{(2)}=(6+4 \sqrt{3}) / 27, \quad a_{22}^{(2)}=(3-\sqrt{3}) / 27 \text {. }
\end{aligned}
$$

As mentioned in Section 3, this discretization of (3.1) is characterized by $r_{0}=2 m-$ $1=3, r_{1}=2 m=4$, and hence by $r=\min \left(r_{0}+1, r_{1}\right)=4=2 m$. Since the collocation parameters are the Gauss points we have, in (3.17), $p^{*}=2 m=4$, and thus $q=\min \left(p^{*}, r\right)=4$. The above method is thus optimal.

Example 4.3. $m=2 ; c_{1}=(3-\sqrt{3}) / 6, c_{2}=(3+\sqrt{3}) / 6 ; \tilde{c}_{1}=1 / 3, \tilde{c}_{2}=1($ Radau points for $(0,1]) ; d_{i l}=c_{i} \tilde{c}_{l}$.

The Runge-Kutta parameters are:

$A, b_{1}$ and $b_{2}$ as in Example 4.1:

$$
\begin{aligned}
& D=\left(d_{i l}\right)=\left(\begin{array}{ll}
(3-\sqrt{3}) / 18 & (3-\sqrt{3}) / 6 \\
(3+\sqrt{3}) / 18 & (3+\sqrt{3}) / 6
\end{array}\right) ; \\
& \bar{A}:=\left(\bar{a}_{i l}\right)=\left(\begin{array}{ll}
(3-\sqrt{3}) / 8 & (3-\sqrt{3}) / 24 \\
(3+\sqrt{3}) / 8 & (3+\sqrt{3}) / 24
\end{array}\right) ; \\
& a_{11}^{(1)}=(3+4 \sqrt{3}) / 108, \quad a_{12}^{(1)}=5(3-2 \sqrt{3}) / 108, \\
& a_{21}^{(1)}=1 / 4, \quad a_{22}^{(1)}=(3-2 \sqrt{3}) / 12 ; \\
& a_{11}^{(2)}=5(3+2 \sqrt{3}) / 108, \quad a_{12}^{(2)}=(3-4 \sqrt{3}) / 108, \\
& a_{21}^{(2)}=(3+2 \sqrt{3}) / 12, \quad a_{22}^{(2)}=1 / 4 .
\end{aligned}
$$

Since again we have $r_{0}=2 m-1=3$, and thus by the argument used in Example 4.2 we find, in (3.17), $q=2 m=4$. This method is also optimal.

5. Concluding Remarks. (i) If the domain of definition of the kernel $k(t, s, y)$ in (1.1) can be extended to include values $s>t$, then we may choose, instead of (3.27), $\mu_{0}=\mu_{1}=m, d_{l}=c_{l}, d_{i l}=c_{l}(l=1, \ldots, m ; i=1, \ldots, m)$. If the parameters $\left\{c_{i}\right\}$ are the zeros of $P_{m}(2 s-1)$ (Gauss points) then we have, in analogy to the three examples of Section 4 , three more optimal methods. 
(ii) The block-by-block methods of [12] correspond to special discretizations of (3.1): if the collocation parameters are equally spaced, i.e. if $c_{i}=(i-1) /(m-1)$ $(i=1, \ldots, m)$, and if $d_{i l}=c_{i} c_{l}$, then we have

$$
r_{0}=r_{1}= \begin{cases}m, & \text { if } m \text { is even, } \\ m+1, & \text { if } m \text { is odd. }\end{cases}
$$

Thus, it follows that $r=m$ ( $m$ even), and $r=m+1$ ( $m$ odd), which in turn implies, in (3.17), $q=m$ ( $m$ even), and $q=m+1$ ( $m$ odd). These quadrature formulas are, of course, the formulas of Newton-Cotes.

Institute of Mathematics

University of Fribourg

$\mathrm{CH}-1700$ Fribourg, Switzerland

1. C. T. H. BAKER, "Initial value problems for Volterra integro-differential equations," in Modern Numerical Methods for Ordinary Differential Equations (G. Hall and J. M. Watt, eds.), Clarendon Press, Oxford, 1976, p. 296-307.

2. H. BRUNNER, "On the numerical solution of nonlinear Volterra integro-differential equations," $B I T$, v. 13,1973 , pp. $381-390$.

3. H. BRUNNER, "The application of the variation of constants formulas in the numercial analysis of integral and integro-differential equations," Utilitas Math., v. 19, 1981, pp. 255-290.

4. H. BrunNer \& J. D. LAMBerT, "Stability of numerical methods for Volterra integro-differential equations," Computing, v. 12, 1974, pp. 75-89.

5. C. W. CRYER, "Numerical methods for functional differential equations," in Delay and Functional Differential Equations and Their Applications (K. Schmitt, ed.), Academic Press, New York, 1972, pp. 17-101.

6. A. FeldStein \& J. R. SOPKA, "Numerical methods for nonlinear integro-differential equations," SIAM J. Numer. Anal., v. 11, 1974, pp. 826-846.

7. A. GHIzZETrI \& A. OssicinI, Quadrature Formulae, Birkhäuser Verlag, Basel, 1970.

8. A. Guillou \& J. L. SoulÉ, "La resolution numérique des problemes différentiels aux conditions initiales par des méthodes de collocation," RAIRO Sér. Rouge, v. 3, 1969, pp. 17-44.

9. E. IsAaCson \& H. B. Keller, Analysis of Numerical Methods, Wiley, New York, 1966.

10. C. LUBICH, Numerische Behandlung Volterra'scher Integrodifferentialgleichungen, Diploma Thesis, University of Innsbruck, 1981.

11. C. LuBICH, Runge-Kutta Theory for Volterra Integrodifferential Equations, Preprint No. 131, Sonderforschungsbereich 123, University of Heidelberg, 1981.

12. A. MAKROGLOU, "Convergence of a block-by-block method for nonlinear Volterra integro-differential equations," Math. Comp., v. 35, 1980, pp. 783-796.

13. W. L. MOCARSKY, "Convergence of step-by-step methods for nonlinear Volterra integro-differential equations," J. Inst. Math. Appl., v. 8, 1971, pp. 235-239.

14. S. P. NøRSETT, "Collocation and perturbed collocation methods," in Numerical Analysis, Dundee 1979 (G. A. Watson, ed.), Lecture Notes in Math., Vol. 773, Springer-Verlag, Berlin, Heidelberg, New York, 1980, pp. 119-132.

15. S. P. NøRSETT \& G. WANNER, “The real-pole sandwich for rational approximations and oscillation equations," BIT, v. 19, 1979, pp. 79-94. 